\title{
Solid-state lasers: status and perspectives
}

\author{
G. Huber · J. Limpert • A. Tünnermann
}

Published online: 18 October 2009

(C) Springer-Verlag 2009

Diode-pumped solid-state lasers are attractive sources of coherent radiation for various scientific and technological applications. In the past years these lasers have emerged as attractive power scalable laser concept for continuous wave and pulse operation. However, the different fields of applications increasingly demand more powerful, efficient, and rugged lasers with diffraction limited beam quality in a wide spectral range. Hence, at present a new generation of solidstate lasers is in the focus of the developments. Thin disc and fiber lasers with improved thermo-optical parameters have now entered the regime of multi-kilowatt of average output power with almost diffraction limited beam quality. Wall-plug efficiencies of these advanced solid-state lasers exceed $20 \%$ for systems with emission wavelength in the near infrared even in ultrafast operation. Novel laser materials are applied to broaden the emission characteristics of solid-state lasers to the ultraviolet and infrared spectral range and combined with methods of nonlinear optics for frequency and brightness transformation. In this special issue novel developments in solid-state laser technology are reported, which will have a strong impact on next generation laser systems.

\section{G. Huber}

Institut f. Laser-Physik, Universität Hamburg,

Luruper Chaussee 149, 22761 Hamburg, Germany

e-mail: huber@physnet.uni-hamburg.de

Fax: +49-40-89985190

\section{J. Limpert}

Friedrich Schiller University Jena, Institute of Applied Physics,

Albert-Einstein-Str. 15, 07743 Jena, Germany

e-mail: Jens.Limpert@uni-jena.de

Fax: +49-3641-947802

\section{A. Tünnermann $(\bowtie)$}

Fraunhofer Institute, Applied Optics and Precision Engineering and Friedrich-Schiller Universität Jena, Institut für Angewandte Physik, Albert-Einstein-Strasse 7, 07745 Jena, Germany

e-mail: Andreas.Tuennermann@iof.fraunhofer.de

Fax: +49-3641-807201

url: http://www.iof.fraunhofer.de 